OPEN ACCESS

Edited by:

Mohammed Morad,

Clalit Health Services, Israel

Reviewed by:

Luciana Hannibal,

University Medical Center Freiburg,

Germany

Joav Merrick,

Ministry of Social Affairs, Israel

*Correspondence:

Catherine Qiu Hua Chan catherine.chan.q.h@sgh.com.sg;

Lian Leng Low

low.lian.leng@singhealth.com.sg

Specialty section:

This article was submitted to Family Medicine and Primary Care,

a section of the journal

Frontiers in Medicine

Received: 03 July 2016 Accepted: 09 August 2016 Published: 23 August 2016

Citation:

Chan CQH, Low LL and Lee KH (2016) Oral Vitamin B12 Replacement for the Treatment of Pernicious Anemia.

Front. Med. 3:38.

doi: 10.3389/fmed.2016.00038

\section{Oral Vitamin B12 Replacement for the Treatment of Pernicious Anemia}

\author{
Catherine Qiu Hua Chan ${ }^{1 *}$, Lian Leng Low ${ }^{1,2 *}$ and Kheng Hock Lee ${ }^{1,2}$ \\ ${ }^{1}$ Department of Family Medicine and Continuing Care, Singapore General Hospital, Singapore, ${ }^{2}$ Family Medicine, Duke-NUS \\ Medical School, Singapore
}

Many patients with pernicious anemia are treated with lifelong intramuscular (IM) vitamin B12 replacement. As early as the 1950s, there were studies suggesting that oral vitamin B12 replacement may provide adequate absorption. Nevertheless, oral vitamin B12 replacement in patients with pernicious anemia remains uncommon in clinical practice. The objective of this review is to provide an update on the effectiveness of oral vitamin B12 for the treatment of pernicious anemia, the recommended dosage, and the required frequency of laboratory test and clinical monitoring. Relevant articles were identified by PubMed search from January 1, 1980 to March 31, 2016 and through hand search of relevant reference articles. Two randomized controlled trials, three prospective papers, one systematic review, and three clinical reviews fulfilled our inclusion criteria. We found that oral vitamin B12 replacement at $1000 \mu \mathrm{g}$ daily was adequate to replace vitamin B12 levels in patients with pernicious anemia. We conclude that oral vitamin B12 is an effective alternative to vitamin B12 IM injections. Patients should be offered this alternative after an informed discussion on the advantages and disadvantages of both treatment options.

Keywords: oral vitamin B12, pernicious anemia, mecobalamin, cobalamin, cyanocobalamin

\section{INTRODUCTION}

Vitamin B12 deficiency is a common condition, and many are undiagnosed. Absolute deficiency occur up to $6 \%$ of those aged 60 years and older, whereas marginal deficiency occur in close to $20 \%$ of patients in later life (1). The manifestation of Vitamin B12 deficiency ranges from subtle, non-specific clinical features to serious neurological and neuropsychiatric complication if left untreated. With an aging population, screening for vitamin B12 level as part of anemia and cognitive impairment workup is more common. More cases are diagnosed, resulting in rising incidence of patients with vitamin B12 deficiency. The common causes of vitamin B deficiency are food-cobalamin (vitamin B12) malabsorption and pernicious anemia.

Pernicious anemia is an autoimmune gastritis resulting from the destruction of gastric parietal cells and consequent impairment of intrinsic factors secretion to bind the ingested vitamin B12. Other autoimmune disorders, especially thyroid disease, diabetes mellitus, and vitiligo, are also commonly associated with pernicious anemia. The cost and availability of auto-antibodies testing, such as intrinsic factor and anti-parietal cell antibodies, can be a barrier to further investigation for vitamin B12 deficiency to exclude pernicious anemia. Therefore, the exact prevalence of pernicious anemia is difficult to ascertain. It has been estimated that the prevalence of pernicious anemia in European countries is approximately $4 \%$ of the population (2). It is also well acknowledged that the prevalence increase with age and therefore more common in the elderly.

Abbreviation: RCT, randomized controlled trial. 
For patients with pernicious anemia, lifelong vitamin B12 therapy is indicated. Vitamin B12 is absorbed in the terminal ileum. This absorption is almost entirely dependent on intrinsic factor binding to vitamin B12. This bound complex in turn binds to the cubam receptor in the terminal ileum and is internalized. The complex is eventually released from lysosomes and transported across the cell membrane bound to transcobalamin in the blood circulation. Traditionally, vitamin B12 replacement is administered intramuscularly. However, it is believed that oral vitamin B12 can be absorbed passively independent of intrinsic factors. Passive diffusion accounts for about $1 \%$ of total absorption, and this route of absorption is unaffected in patients with pernicious anemia (3).

There were studies in the 1950s and the1960s that showed that oral vitamin B12 could be absorbed by patients with pernicious anemia and could lead to resolution of the anemia (3-6). However, lifelong intramuscular (IM) injection for replacement is still a common practice. In 1991, a survey done on Minneapolis internists led one commentary to conclude that oral vitamin B12 replacement for pernicious anemia was one of "medicine's best kept secret" (7). In 1996, when the survey was repeated again in the same area, awareness and use of oral vitamin B12 for pernicious anemia had increased substantially (0-19\%), but the majority of doctors still remain unaware of this treatment option $(61 \%)(8)$.

The objective of our review is to inform clinicians on the effectiveness of oral vitamin B12 as adequate replacement in patients with pernicious anemia, as well as make recommendations on the dosing and frequency of clinical and laboratory monitoring.

\section{METHODS}

A PubMed search was conducted in April 2016 to identify suitable articles published from January 1, 1980 to March 31, 2016. The following search strategy was applied: "Administration, Oral" (MeSH term) AND "Vitamin B12" (MeSH term) AND "Anaemia, Pernicious" (MeSH term). Only studies evaluating the effectiveness of oral vitamin B12 replacement on pernicious anemia patients in entirety or as a subset were included for review. The search was limited to English articles.

The search strategy was summarized in the following flow chart.

\section{Flowchart on Selection of Articles}

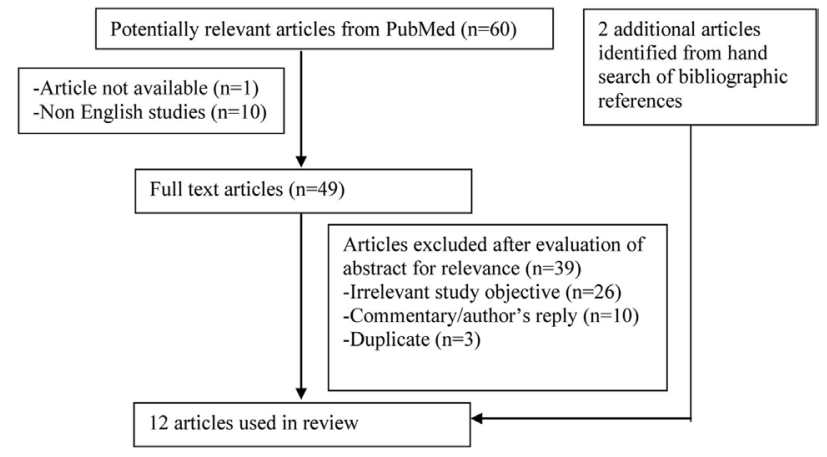

Grading of level of evidence and strength of recommendation was based on strength of recommendation taxonomy (SORT) framework (9). The description and level of evidence of included studies are shown in Table 1.

\section{RESULTS}

Sixty articles were identified through the electronic database search. Non-English studies were excluded. The abstracts of the remaining 49 articles were evaluated for relevance. Articles that did not involve or discuss about patients with pernicious anemia treated with oral vitamin B12 replacement were excluded. Duplicated articles and commentary/author's reply were excluded. Another two articles were identified from hand search of bibliographic references of the shortlisted articles. A total of 12 articles [2 randomized controlled trials (RCTs) $(10,11), 3$ prospective studies $(12-14), 1$ systematic review (15), and 6 clinical reviews $(2,16-20)]$ were obtained for the review.

\section{Effectiveness of Oral Vitamin B12}

The data given in the studies all supported the use of oral vitamin B12 as a valid and effective way of treating vitamin B12 deficiency, including pernicious anemia. The age range of the study population was 23-92 years old. The RCT by Kuzminski et al. (10) and prospective study by Delpre et al. (12) were done in America. The RCT by Bolaman et al. (11) was done in Turkey. The other two prospective studies by Nyholm et al. (13) and Andres et al. (14) were done in United Kingdom and France, respectively. The studies were conducted during the period of late 1990s to early 2000s.

In Kuzminski's study, serum vitamin B12 levels were significantly higher in the oral (vitamin B12 $2000 \mu \mathrm{g}$ ) compared with IM (vitamin B12 $1000 \mu \mathrm{g})$ group $(643 \pm 328$ vs. $306 \pm 118 \mathrm{pg} /$ $\mathrm{mL} ; p<0.001)$ at 2 months. The difference was even greater at 4 months $(1005 \pm 595$ vs. $325 \pm 165 \mathrm{pg} / \mathrm{mL})$. Five of the patients who had pernicious anemia in the oral vitamin B12 replacement group all had increase in serum vitamin B12 level. Four of the 18 in the oral group and 4 of the 15 in the IM group had a neurological response with a marked improvement or clearing of paresthesia, ataxia, or memory loss.

For Bolaman's study, there was also an increase in serum vitamin B12 levels in both groups (oral vitamin B12 $1000 \mu \mathrm{g}$ vs. IM vitamin B12 $1000 \mu \mathrm{g}$ ) at 90 days (Oral group $213.8 \mathrm{pg} / \mathrm{mL}$ and IM group $225.5 \mathrm{pg} / \mathrm{mL}$ ). There was a statistically significant difference between days 0 and 90 in both groups $(p<0.0001)$, but authors did not analyze difference between both groups. Both groups reported improvements of cognitive functions, sensory neuropathy, and vibration sense, but there was no statistical significant difference between both groups.

The systematic review by Butler et al. (15) done on these two RCTs concluded that high oral doses of vitamin B12 could be as effective as IM administration in achieving short-term hematological and neurological responses. However, the two RCTs were limited by their small sample size and short follow-up period.

The rest of studies also had small sample size but some had longer follow-up period (up to 18 months). Normalization of serum vitamin B12 levels was seen in all patients (inclusive of patients with pernicious anemia) in Delpre's study. An increase 
TABLE 1 | Description and level of evidence for articles reviewed.

\begin{tabular}{|c|c|c|c|c|}
\hline $\begin{array}{l}\text { Study and study } \\
\text { type }\end{array}$ & $\begin{array}{l}\text { Participants, sample } \\
\text { size, follow-up duration }\end{array}$ & Intervention, outcome measure & Results & $\begin{array}{l}\text { Level of } \\
\text { evidence } \\
\text { (based on } \\
\text { SORT) }\end{array}$ \\
\hline
\end{tabular}

Kuzminski et al. Newly diagnosed vitamin (10), randomized B12-deficient patients

(RCT), not blinded controlled trial

Oral vitamin B12 $2000 \mu \mathrm{g}$ daily for 120 days vs. intramuscular (IM) vitamin B12 $1000 \mu \mathrm{g}$ on days $1,3,7,10,14,21,30,60$, and 90

Primary outcomes: serum vitamin B12, methylmalonic acid, homocysteine neurologic responses

pernicious anemia)

Control = 15 (2 had

pernicious anemia)

120 days

Bolaman et al.

(11), RCT, not

blinded

Megaloblastic anemia due to vitamin B12 deficiency

Oral vitamin B12 $1000 \mu \mathrm{g}$ daily for 90 days vs. IM vitamin B12 $1000 \mu \mathrm{g}$ daily for 10 days, then once weekly for 28 days and after that
Intervention $=26(8 \mathrm{had}$ presence of anti-parietal call antibody)

Control $=34$ ( 3 had

presence of anti-parietal

call antibody)

90 days

Delpre et al. (12), Vitamin B12 deficiency

Prospective,

open-label

$N=18$ (inclusive of patients with pernicious anemia but did not state number)

7-12 days

Nyholm et al. (13), Vitamin B12 deficiency Prospective, case series

$N=40$ (10 patients had pernicious anemia)

3-18 months

Andres et al. Pernicious anemia

(14), Prospective, $\quad N=10$

open-label 3 months continued with once monthly

Primary outcomes: serum vitamin B12, hemoglobin, platelet count, MCV, WBC, mini-mental state examination, neurological assessment

Sublingual vitamin B12 $1000 \mu \mathrm{g}$ daily for 7-12 days

Primary outcome: serum vitamin B12

Loading dose of IM vitamin B12 till vitamin B12 level reached lower 25th centile $(418 \mathrm{pg} /$ $\mathrm{mL}$ ) and then converted to oral vitamin B12 $1000 \mu \mathrm{g}$ daily

Primary outcomes: serum vitamin B12, hemoglobin, MCV, homocysteine, and neurological assessment

Oral vitamin B12 $1000 \mu \mathrm{g}$ daily for 3 months Primary outcome: serum vitamin B12, secondary outcomes: hemoglobin, platelet count, and MCV
Serum vitamin B12 levels were significantly higher in the oral compared with IM group $(643 \pm 328$ vs. $306 \pm 118 \mathrm{pg} / \mathrm{mL} ; p<0.001)$ at 2 months. The difference was even greater at 4 months (1005 \pm 595 vs. $325 \pm 165 \mathrm{pg} / \mathrm{mL}$ )

Four of the 18 in the oral group and 4 of the 15 in the IM group had a neurological response with a marked improvement or clearing of paresthesia, ataxia, or memory loss

Serum vitamin B12 levels increased in both groups at 90 days (oral group $213.8 \mathrm{pg} / \mathrm{mL}$ and IM group $225.5 \mathrm{pg} / \mathrm{mL}$ ). There was a statistically significant difference between day 0 and day 90 in both groups $(p<0.0001)$ but authors did not analyze difference between both groups Both groups reported improvements of cognitive functions, sensory neuropathy, and vibration sense, but there was no statistical significant difference between both groups

Normalization of serum vitamin B12 levels was seen in all patients. An increase in vitamin B12 level was as much as fourfold compared with pretreatment in most patients. The mean change of $387.7 \mathrm{pg} / \mathrm{mL}$ was statistically significant $(p=0.0001$, Student's t-test)

2

2

Oral vitamin B12 was effective in all the patients (no patients had to restart IM vitamin B12). At 3 months, the median serum vitamin B12 level was $1193 \mathrm{pg} / \mathrm{mL}$

Oral treatment did not result in any new neurological complications

After 3 months, serum vitamin B12 levels were $\mathrm{mL} ; p<0.001$ ). One patient's result was not available due to technical problem in vitamin B12 level was as much as fourfold compared with pretreatment in most patients. The mean change of $387.7 \mathrm{pg} /$ $\mathrm{mL}$ was significant ( $p=0.0001$ in Student's $t$-test). Oral vitamin $\mathrm{B} 12$ was effective in all the patients (10 patients had pernicious anemia) in Nyholm's study with the median serum vitamin B12 level of $1193 \mathrm{pg} / \mathrm{mL}$ after 3 months of treatment. It was also reported that using oral treatment did not result in any new neurological complications. Andres's study was the only study done on patients with pernicious anemia in entirety. All nine patients' serum vitamin B12 levels improved (mean increase, $117.4 \mathrm{pg} / \mathrm{mL}$; $p<0.001$ ) after 3 months.

\section{Dosage of Oral Vitamin B12 Replacement Required}

In all the five studies, an oral (Delpre's study via sublingual route) dose of $1000 \mu \mathrm{g}$ vitamin B12 was used with the exception of Kuzminski's study, whereby a higher dose of $2000 \mu \mathrm{g}$ was used. It had been showed that oral vitamin B12 at $1000 \mu \mathrm{g}$ was adequate replacement in pernicious anemia patients.

There was a dose-finding trial done by Eussen et al. (21), and the results indicated that the lowest dose of oral vitamin B12 required to normalize biochemical markers of mild vitamin B12 deficiency in older people was more than 200 times greater 
than the recommended dietary allowance for vitamin B12 of approximately $3 \mu \mathrm{g} /$ day. However, this study did not distinguish the extent to which differences in individual responses were due to active as opposed to passive absorption of vitamin B12.

In some of the clinical reviews, it was stated that many do not use oral vitamin B12 replacement in view of concern on the unpredictable absorption at low doses of oral replacement. Daily vitamin B12 turnover rate is about $2 \mu \mathrm{g} /$ day, so an oral dose of $100-250 \mu \mathrm{g} /$ day is sufficient for normal patients. However, in view of the estimated $1 \%$ of total absorption via passive diffusion in patients with pernicious anemia, a $1000 \mu \mathrm{g}$ daily dose is recommended.

\section{Frequency of Monitoring}

The outcome measurement of vitamin B12 level was done from a range of 1-3 months (exception of Delpre's study, which was done ranging from 9 to 14 days). Improvement in vitamin B12 was seen as early as within a month. Only in Nyholm's study, 11 of their patients were followed up until 18 months, whereby the vitamin B12 levels were maintained.

Close monitoring monthly is necessary at the start of oral replacement to verify normalization of lab results and monitoring for symptoms. Thereafter, annual monitoring should suffice. It should be carried out on a regular interval that is safe for patients and acceptable to both patients and doctors.

\section{DISCUSSION}

We summarized the level of evidence of using oral vitamin B12 replacement for patients with pernicious anemia (Table 1). The strength of clinical recommendations based on the SORT framework is provided in Table 2.

There is no "gold standard" in testing for vitamin B12 deficiency. It has been recommended that serum total homocysteine (Hcy) and methylmalonic acid (MMA) levels are more sensitive indicators of vitamin B12 status in pernicious anemia patients without any other disorders of vitamin B12 metabolism. In our review, some $(10,13)$, but not all, studies determine metabolite levels (Hcy and MMA) in pernicious anemia to assess effectiveness of therapy with vitamin B12. Most patients with pernicious

TABLE 2 | SORT recommendations for clinical practice.

\begin{tabular}{lc}
\hline Clinical recommendation & $\begin{array}{c}\text { Strength of } \\
\text { recommendation }\end{array}$
\end{tabular}

Oral vitamin B12 can be used for adequate replacement in B

patients with pernicious anemia

An oral vitamin B12 dose at $1000 \mu \mathrm{g}$ is adequate replacement in patients with pernicious anemia

B

Close monitoring monthly is necessary at the start of oral replacement to verify normalization of lab results and monitoring for symptoms

Thereafter, annual monitoring should suffice

Elevated serum homocysteine and methylmalonic acid levels should be included in future assessments of pernicious anemia and corrected to normal levels in patients with pernicious anemia anemia are not screened genetically for confirmatory causes of the disease. There are genetic errors of metabolism where serum vitamin B12 levels are normal, but there is a functional (cellular) level deficiency of the micronutrient that is often overlooked unless Hcy and MMA levels are also measured. Both Hcy and MMA levels are elevated in patients with vitamin B12 deficiency. Elevated serum Hcy and MMA levels (>3 SDs above the mean in normal subjects) have a sensitivity of 95.9 and $98.4 \%$, respectively, to diagnose vitamin B12 deficiency (22). The levels decrease immediately after treatment and repeat measurements have clinical utility to document adequate vitamin B12 replacement. However, considerations of using Hcy and MMA levels would include cost, availability of the test, as well as having standardized reference intervals.

Patients with vitamin B12 deficiency who are symptomatic have severe neurological deficits or have critically low blood levels of vitamin B12 should be treated with IM administration. This is to ensure rapid replenishment of body stores to prevent irreversible consequences of deficiency. Subsequently, patients may be able to convert to oral replacement with close monitoring. For long-term maintenance therapy, oral vitamin B12 replacement can be effective in patients with pernicious anemia. Patient preference should be taken into consideration in the choice of treatment options. Few studies had included surveying patients regarding preference of choice between oral vs. IM replacement of vitamin B12. In Delpre's study, $87 \%$ of them preferred tablets to injection (12). Eighty-seven percent found the tablets highly acceptable, while the remaining $13 \%$ agreed that tablets were acceptable. It must also be considered that adherence is likely to be better if the patient's preferred route of administration is taken into consideration.

Additional factors to consider when helping patient to make an informed choice are as follows.

\section{Cost}

In a study done in United Kingdom by Vidal-Alaball et al. (23), using oral vitamin B12 after diagnosis or switching from IM route could save resources in the medium and long term. The use of oral route results in significant reduction in manpower costs.

\section{Reduction of Scheduled Visits to Clinics}

Many patients with vitamin B12 deficiency are elderly and have multiple co-morbidities. They often have multiple appointments to attend various clinics and may have frequent hospitalization episodes. The need to schedule vitamin B12 injections is an avoidable addition to the cost and complexity of their care.

\section{Adherence}

In patients with non-compliance to oral medication, IM route may be a better option to ensure timely administration. On the other hand, oral replacement may improve adherence for patients who prefer oral medication to injections.

\section{Discomfort/Pain}

Oral replacement will be useful in patients who are averse to injection. For elderly patients with sarcopenia, injections can painful and difficult to administer. 


\section{Risk Associated with IM Injection}

In patients, whereby IM injections are contraindicated because of coagulopathy or the use anti-coagulation/anti-platelet medication. Oral replacement is the best option.

\section{LIMITATIONS}

1. The review was conducted only using PubMed and hand search.

2. Studies were mainly on patients with vitamin B12 deficiency with a subset of patients with pernicious anemia. Therefore, the actual sample size of pernicious anemia patients in each study may not reach statistical significance. We overcome this by triangulating our conclusions and recommendations based on the findings from multiple studies and the consistent oral vitamin B12 dosage of $1000 \mu \mathrm{g}$.

3. Many of the studies had small sample size and assessed only short-term outcomes. The long-term efficacy and side effects require further evaluation.

4. A few possible relevant articles were excluded due to language issues.

\section{DIRECTION OF FUTURE RESEARCH}

A multicenter randomized clinical trial is current in progress in Spain primary health-care setting called Project OB12 (24). This study aims to provide a more conclusive answer with a large sample size of 320 patients and longer follow-up period of 52 weeks. Further studies should include testing the efficacy of different

\section{REFERENCES}

1. Allen LH. How common is vitamin B-12 deficiency? Am J Clin Nutr (2009) 89(2):693S-6S. doi:10.3945/ajcn.2008.26947A

2. Stabler SP. Clinical practice. Vitamin B12 deficiency. N Engl J Med (2013) 368(2):149-60. doi:10.1056/NEJMcp1113996

3. Berlin H, Berlin R, Brante G. Oral treatment of pernicious anemia with high doses of vitamin B12 without intrinsic factor. Acta Med Scand (1968) 184(4):247-58. doi:10.1111/j.0954-6820.1968.tb02452.x

4. Withey JL, Jones JH, Kilpatrick GS. Long-term trial of oral treatment of pernicious anaemia with vitamin-B12-peptide. Br Med J (1963) 1(5345):1583-5. doi:10.1136/bmj.1.5345.1583

5. Meyer LM, Sawitsky A, Cohen BS, Krim M, Fadem R. Oral treatment of pernicious anemia with vitamin B12. Am J Med Sci (1950) 220(6):604-9. doi:10.1097/00000441-195022060-00002

6. Ungley CC. Absorption of vitamin B12 in pernicious anaemia. I. Oral administration without a source of intrinsic factor. $\mathrm{Br}$ Med J (1950) 2(4685):905-8. doi:10.1136/bmj.2.4685.908

7. Lederle FA. Oral cobalamin for pernicious anemia. Medicine's best kept secret? JAMA (1991) 265(1):94-5. doi:10.1001/jama.265.1.94

8. LederleFA. Oral cobalamin for pernicious anemia: back from the verge of extinction. J Am Geriatr Soc (1998) 46(9):1125-7. doi:10.1111/j.1532-5415.1998. tb06651.x

9. Ebell MH, Siwek J, Weiss BD, Woolf SH, Susman J, Ewigman B, et al. Strength of recommendation taxonomy (SORT): a patient-centered approach to grading evidence in the medical literature. Am Fam Physician (2004) 69(3):548-56.

10. Kuzminski AM, Del Giacco EJ, Allen RH, Stabler SP, Lindenbaum J. Effective treatment of cobalamin deficiency with oral cobalamin. Blood (1998) 92(4):1191-8. dosages. It may be important to study the knowledge and practices of doctors/health-care workers with regard to oral replacement therapy with vitamin B12 for patients with pernicious anemia. Surveys on patients' preferences for oral or IM replacement would be informative to guide clinical decision-making.

\section{CONCLUSION}

Oral vitamin B12 replacement at $1000 \mu \mathrm{g}$ daily is an adequate alternative to IM B12 injections. Close monitoring with clinical review and repeat vitamin B12 levels are required on a monthly basis to review symptoms and ensure normalization of B12 deficiency. Elevated serum Hcy and MMA levels should be included in future assessments of pernicious anemia and corrected with normal levels in patients with pernicious anemia.

\section{AUTHOR CONTRIBUTIONS}

Conceived and designed the study: CC, LL, and KL. Performed the study: CC and LL. Analyzed the data: CC and LL. Interpreted the results: CC and LL. Wrote the paper: CC, LL, and KL. Principal Investigator of this study and supervised this study: CC. Revised the paper critically and give final approval for publication: all authors.

\section{FUNDING}

This is an investigator-initiated study, and no grant funding was obtained.

11. Bolaman Z, Kadikoylu G, Yukselen V, Yavasoglu I, Barutca S, Senturk T Oral versus intramuscular cobalamin treatment in megaloblastic anemia: a single-center, prospective, randomized, open-label study. Clin Ther (2003) 25(12):3124-34. doi:10.1016/S0149-2918(03)90096-8

12. Delpre G, Stark P, Niv Y. Sublingual therapy for cobalamin deficiency as an alternative to oral and parenteral cobalamin supplementation. Lancet (1999) 354(9180):740-1. doi:10.1016/S0140-6736(99)02479-4

13. Nyholm E, Turpin P, Swain D, Cunningham B, Daly S, Nightingale P, et al. Oral vitamin B12 can change our practice. Postgrad Med J (2003) 79(930):218-20. doi:10.1136/pmj.79.930.218

14. Andres E, Henoun Loukili N, Noel E, Maloisel F, Vinzio S, Kaltenbach G, et al. Effects of oral crystalline cyanocobalamin $1000 \mathrm{mug} / \mathrm{d}$ in the treatment of pernicious anemia: an open-label, prospective study in ten patients. Curr Ther Res Clin Exp (2005) 66(1):13-22. doi:10.1016/j.curtheres.2005. 02.001

15. Butler CC, Vidal-Alaball J, Cannings-John R, McCaddon A, Hood K, Papaioannou A, et al. Oral vitamin B12 versus intramuscular vitamin B12 for vitamin B12 deficiency: a systematic review of randomized controlled trials. Fam Pract (2006) 23(3):279-85. doi:10.1093/fampra/ $\mathrm{cml} 008$

16. Shipton MJ, Thachil J. Vitamin B12 deficiency - a 21st century perspective. Clin Med (Lond) (2015) 15(2):145-50. doi:10.7861/clinmedicine.15-2-145

17. Andres E, Serraj K. Optimal management of pernicious anemia. J Blood Med (2012) 3:97-103. doi:10.2147/JBM.S25620

18. Oh R, Brown DL. Vitamin B12 deficiency. Am Fam Physician (2003) 67(5):979-86.

19. Lane LA, Rojas-Fernandez C. Treatment of vitamin B(12)-deficiency anemia: oral versus parenteral therapy. Ann Pharmacother (2002) 36(7-8):1268-72. doi:10.1345/aph.1A122 
20. Paauw DS. Did we learn evidence-based medicine in medical school? Some common medical mythology. J Am Board Fam Pract (1999) 12(2):143-9. doi:10.3122/jabfm.12.2.143

21. Eussen SJ, de Groot LC, Clarke R, Schneede J, Ueland PM, Hoefnagels WH, et al. Oral cyanocobalamin supplementation in older people with vitamin B12 deficiency: a dose-finding trial. Arch Intern Med (2005) 165(10):1167-72. doi:10.1001/archinte.165.10.1167

22. Savage DG, Lindenbaum J, Stabler SP, Allen RH. Sensitivity of serum methylmalonic acid and total homocysteine determinations for diagnosing cobalamin and folate deficiencies. Am JMed (1994) 96(3):239-46. doi:10.1016/0002-9343(94)90149-X

23. Vidal-Alaball J, Butler CC, Potter CC. Comparing costs of intramuscular and oral vitamin B12 administration in primary care: a cost-minimization analysis. Eur J Gen Pract (2006) 12(4):169-73. doi:10.1080/14017430601049449

24. Sanz-Cuesta T, Gonzalez-Escobar P, Riesgo-Fuertes R, GarridoElustondo S, del Cura-Gonzalez I, Martin-Fernandez J, et al. Oral versus intramuscular administration of vitamin B12 for the treatment of patients with vitamin B12 deficiency: a pragmatic, randomised, multicentre, non-inferiority clinical trial undertaken in the primary healthcare setting (project OB12). BMC Public Health (2012) 12:394. doi:10.1186/1471-245812-394

Conflict of Interest Statement: The authors declare that the research was conducted in the absence of any commercial or financial relationships that could be construed as a potential conflict of interest.

Copyright ( $(2016$ Chan, Low and Lee. This is an open-access article distributed under the terms of the Creative Commons Attribution License (CC BY). The use, distribution or reproduction in other forums is permitted, provided the original author(s) or licensor are credited and that the original publication in this journal is cited, in accordance with accepted academic practice. No use, distribution or reproduction is permitted which does not comply with these terms. 